DOI: $10.1515 /$ plass-2018-0002

Grzegorz Żurek $^{*}{ }^{1}$, Kamil Prokopiuk ${ }^{1}$, Danuta Martyniak ${ }^{1}$, Agnieszka Rachwalska ${ }^{2}$, Eugeniusz Paszkowski ${ }^{2}$, Urszula Woźna - Pawlak ${ }^{3}$, Maciej Jurkowski ${ }^{4}$

\begin{abstract}
${ }^{1}$ Plant Breeding and Acclimatization Institute-National Research Institute, Radzików, 05-870 Błonie; ${ }^{2}$ Plant Breeding and Acclimatization Institute, National Research Institute, Experimental Station Grodkowice, 32 - 015 Grodkowice; ${ }^{3}$ DANKO Hodowla Roślin sp. z o.o., 64 - 000 Kościan; ${ }^{4}$ Poznańska Hodowla Roślin, sp. z o.o., 63-004 Tulce; ${ }^{5}$ Małopolska Hodowla Roślin, sp. z o.o., 30-002 Kraków; *Corresponding author's e-mail: g.zurek@ihar.edu.pl
\end{abstract}

\title{
SEED YIELD AND ITS COMPONENTS IN THREE FESTUCA SPECIES
}

\section{ABSTRACT}

An experiment has been performed in four locations in Poland (Radzików, Leszno, Szelejewo and Nieznanice). Fifteen genotypes from three species (tall fescue - Festuca arundinacea, meadow fescue - F. pratensis and red rescue - F. rubra) were measured and observed during two consecutive years. Despite of phenological observations (heading and flowering start dates), biometrical measurements (plant height, leaf dimension, number of generative stems etc.) and physiological trait (chlorophyll contents index) seed yield of single panicle, seed yield of plant and seed yield per plot (i.e. 50 plants) were determined.

Significant effects of years, locations and genotypes were calculated for mentioned species for almost all tested traits. Multiple linear regression analysis was used to estimate major predictors of seed yield. It resulted in different numbers of predictors for different species. For meadow fescue five, for red fescue - three and for tall fescue - only two statistically significant predictors were selected. For all species leaf width and number of generative stems were statistically significant and had positive weights. For meadow and red fescue - also seed yield from single inflorescence was significant with positive weight. Our results demonstrate that leaf width together with number of steams and single panicle yield are the most important determinants of plant seed yield in three Festuca species.

Key words: meadow fescue, leaf, multiple regression analysis, red fescue, tall fescue

\section{INTRODUCTION}

Seed production is one of the important steps in plant breeding since the commercial value of cultivar is often determined by its seed yield capacity (Rognli 2007). Although seed yield is a complex trait and affected by agricultural practices as well as environmental factors, traits related to seed

Communicated by Dariusz Mańkowski 
production reveal considerable genetic variation, prerequisite for improvement by direct or indirect selection (Boelt and Studer, 2010). Numerous research documented primary, secondary etc. traits with its quantified relation to seed yield in most of grass species significant for agricultural practice (Hill and Watkin 1975, Griffiths et al. 1980, Elgersma 1990, Faire and Lefkovitch 1999, Fang 2004, Wang et al. 2011; Biligetu et al. 2012, Martyniak et al. 2017). However, little selection has been exerted on seed yield and its components in forage grasses, therefore genetic variation and heritability for these traits are relatively large (Rognli, 2007, Rognli et al. 2010).

From numerous different grass species, fescues are a group of very diverse cool season perennial grasses (ca. 450 species worldwide) from which some species are commercially and agronomical valued for wide range of applications: from forage, turf, landscape up to ornamental or even bioenergy purposes. Generally, 170 species and more than 50 subspecies from genus Festuca has been described only in European flora (Markgraf - Dannenberg, 1980). However, only for less than 10 species practical applications has been defined and cultivars has been bred.

Three following species are currently of the wider application: meadow fescue (Festuca pratensis Huds.), tall fescue (Festuca arundinacea Schreb.) and red fescue (Festuca rubra L.). Meadow fescue is loosely tufted grass, mainly used for forage and pasture. It is considered native to Europe and Eurasia and is one of the most widely used forage grasses in the Nordic area due to its superior combination of fodder quality and winter hardiness (Fjellheim et al. 2006; 2007; Rognli et al. 2010). Meadow fescue is morphologically related to tall fescue for which it is mentioned as one of progenitors (Sleper and West, 1996). Tall fescue is tall-growing, erect, stout and tufted grass. It is considered to be the most important forage species of the genus Festuca throughout the temperate regions of the world. It is also grown for lawns, turf, conservation as well as for bioenergy or paper industry (Meyer and Watkins 2003; El Bassam 2010; Danielewicz et. al. 2015, Lalak et al. 2016). Red fescue is very common in native habitats in Europe, Asia and North America where it grows on permanent grasslands used mainly for forage. Red fescue is fine leaved, loosely caespitose, usually long rhizomatous and creeping grass, grown for forage, lawn and turf (Ruemmele et al. 2003).

Seed production is a key feature of any plants reproduced generatively. Even top quality of forage or turf has no practical application without seed produced on reasonable costs. Increase in grass seed production depends on many variables, where breeding potential is still one of the most effective 'drivers' of seed production efficiency. The basic factors contributing to seed yield in grasses are the number of inflorescences produced per plant, the number of florets produced per inflorescence (or head size), the proportion of florets which set seed (or seed setting) and individual seed weight (Griffiths et al. 1980). Fang et al. (2004) proved that the overriding component trait determining meadow fescue plant seed yield is the panicle fertility. And since it is highly correlated with seed weight per panicle, the latter 
could be used in selection for seed production instead of panicle fertility since it is easier to measure (Fang et al. 2004). No such information are currently available is it also true for red and tall fescue. Therefore, to improve our knowledge on the main seed yield components in major Festuca species, experiment has been established in 2014 to define relations between the set of phenotypic characters measured and observed in three Festuca species with plant seed yield.

\section{MATERIAL AND METHODS}

Experiment was established in four locations in Poland: Radzików, Szelejewo, Leszno and Nieznanice. Fifteen genotypes from three Festuca species were used, including commercial cultivars for each species (Table 1).

List of genotypes used in experiment, origin of seed accessions and thousand seed weight

Table 1

\begin{tabular}{|c|c|c|c|c|}
\hline Species & No. & $\begin{array}{l}\text { Name of cultivar or } \\
\text { genotype number }\end{array}$ & Sample origin & TSW $[\mathrm{g}]$ \\
\hline \multirow{5}{*}{$\begin{array}{c}\text { Festuca } \\
\text { pratensis }\end{array}$} & 1 & Pasja & cultivar, provided by the breeder (Bartążek PB)* & 1,86 \\
\hline & 2 & $49-8 b$ & Ecotype & 2,14 \\
\hline & 3 & POB-S-84 & breeding strain, (Małopolska PB) & 1,99 \\
\hline & 4 & POB-S-89 & breeding strain, (Małopolska PB) & 2,37 \\
\hline & 5 & POB-S-91 & breeding strain, (Małopolska PB) & 1,83 \\
\hline \multirow{5}{*}{$\begin{array}{c}\text { Festuca } \\
\text { rubra }\end{array}$} & 1 & Areta & cultivar provided by the breeder (Poznańska PB) & 1,44 \\
\hline & 2 & $109-2 / 1$ & breeding strain, PB\&AI, Radzików ** & 0,92 \\
\hline & 3 & NIB - 289 & breeding strain, (Małopolska PB) & 1,17 \\
\hline & 4 & NIB - 231 & breeding strain, (Małopolska PB) & 0,63 \\
\hline & 5 & NIB - 304 & breeding strain, (Małopolska PB) & 1,09 \\
\hline \multirow{5}{*}{$\begin{array}{l}\text { Festuca } \\
\text { arundina- } \\
\text { cea }\end{array}$} & 1 & Rahela & cultivar provided by the breeder (Bartążek PB) & 3,39 \\
\hline & 2 & $121-2 / 8$ & genotype from experiments, PB\&AI, Radzików & 2,60 \\
\hline & 3 & $124-1 / 8$ & genotype from experiments, PB\&AI, Radzików & 2,20 \\
\hline & 4 & $127-1 / 1$ & genotype from experiments, PB\&AI, Radzików & 1,81 \\
\hline & 5 & $128-1 / 6$ & genotype from experiments, PB\&AI, Radzików & 2,31 \\
\hline
\end{tabular}

Explanation: * - PB - Plant Breeding, ** - Plant Breeding and Acclimatization Institute, Radzików

Before the experiment set-up, six representative soil samples per one location were collected at spring 2014. Soil sampling was made according to PN-ISO 10381-2:2007P. Soil analysis were further performed at Regional Agrochemical Station in Warszawa (Table 2). Soils in experiment locations were poor in soil organic matter $(1.07-1.37 \%)$ but rich in sand fractions and were classified as sandy loam (Radzików, Szelejewo and Nieznanice) or loamy sand (Leszno). Soil pH was acid in Radzików, and slightly acid in other locations. Soil in Radzików was poor in nitrogen contents (especially $\mathrm{N}_{-} \mathrm{NO}_{3}$ ) but rich in $\mathrm{P}$ and $\mathrm{K}$. Despite of observed differences in macro- and micronutrients contents, further management was similar in all locations. 
Soil characteristics at each location and results of analysis of variance between locations (significance of difference: ns - not significant, **** for $\mathrm{P}>99.9 \%$, ** - for $99.9 \%>P>95 \%$ )

\begin{tabular}{|c|c|c|c|c|c|}
\hline Soil parameter: & Radzików & Szelejewo & Leszno & Nieznanice & $\begin{array}{l}\text { Significance of } \\
\text { difference }\end{array}$ \\
\hline Coarse sand $(2.0-0,5 \mathrm{~mm})$ & 53.52 & 68.53 & 64.37 & 71.86 & $* * *$ \\
\hline Medium sand $(0.5-0.02)$ & 24.33 & 12.42 & 10.93 & 14.45 & $* * *$ \\
\hline Silt $(0.02-0.002 \mathrm{~mm})$ & 18.63 & 16.04 & 11.22 & 11.85 & $* *$ \\
\hline Clay $(<0.002 \mathrm{~mm})$ & 3.50 & 3.01 & 1.81 & 1.84 & $* * *$ \\
\hline SOM [\%] & 1.11 & 1.07 & 1.13 & 1.37 & $* *$ \\
\hline Salinity $[\mathrm{g} / \mathrm{KCl}]$ & 0.15 & 0.25 & 0.25 & 0.18 & $* *$ \\
\hline $\mathrm{pH}$ & 5.89 & 6.60 & 6.39 & 6.30 & $* *$ \\
\hline \multicolumn{6}{|c|}{$\left[\mathrm{mg} \times \mathrm{kg}^{-1}\right.$ of soil]: } \\
\hline $\mathrm{N}-\mathrm{NO}_{3}$ & 9.00 & 15.67 & 16.33 & 12.00 & $* * *$ \\
\hline $\mathrm{N}-\mathrm{NH}_{4}$ & 14.17 & 10.17 & 11.50 & 12.00 & $* *$ \\
\hline $\mathrm{N}-$ total & 23.17 & 25.83 & 27.83 & 24.00 & $* *$ \\
\hline $\mathrm{P}$ & 149.83 & 64.67 & 79.67 & 89.50 & $* * *$ \\
\hline $\mathrm{K}$ & 151.67 & 95.33 & 116.17 & 94.67 & $* * *$ \\
\hline $\mathrm{Ca}$ & 634.17 & 623.17 & 573.67 & 589.50 & ns \\
\hline $\mathrm{Mg}$ & 58.67 & 127.83 & 97.33 & 119.17 & $* * *$ \\
\hline $\mathrm{Cl}$ & 12.33 & 14.67 & 18.67 & 15.50 & $* *$ \\
\hline $\mathrm{Cu}$ & 2.52 & 2.47 & 2.63 & 0.97 & $* *$ \\
\hline $\mathrm{Fe}$ & 71.65 & 88.12 & 68.45 & 37.77 & $* *$ \\
\hline $\mathrm{Mn}$ & 7.40 & 2.32 & 3.27 & 4.62 & $\mathrm{~ns}$ \\
\hline $\mathrm{Zn}$ & 5.22 & 5.70 & 4.30 & 6.40 & $* *$ \\
\hline $\mathrm{B}$ & 0.02 & 0.02 & 0.08 & 0.12 & $* *$ \\
\hline
\end{tabular}

At each location plants were planted in the field at spring 2014 in three replication, 50 plants per replication. Plants were planted in rows, $0.25 \mathrm{~cm}$ between plants in row and $0.75 \mathrm{~cm}$ between rows. Fertilization was applied in spring $\left(60 \mathrm{~kg} \mathrm{~N} \times \mathrm{ha}^{-1}\right)$ and in autumn $\left(80 \mathrm{~kg}\right.$ of $\mathrm{P}$ and $\left.\mathrm{K} \times \mathrm{ha}^{-1}\right)$ each year in each location. Mentioned research was conducted during two years: 2015 and 2016.

Following traits were evaluated each year in each experiment location:

- Overwintering (OW), in scale $1-9$, where 1 - plants completely destroyed, 9 - plants vigorous,

- $\quad$ Plant growth habit $(\mathrm{GH})$, in scale $1-9$, where 1 - plants completely flat, 9 - plants erect;

- $\quad$ Time of inflorescence emergence / mean heading start date (HE), expressed in number of days from the 1-st of April to that moment when on $30 \%$ of spaced plants on plot 'emerged' tillers were visible (not less than 3 tillers per plant). Emerged tillers were noted when part of head past base of flag leaf.

- Mean flowering start date (FE), expressed in number of days from the 1 -st of April to that moment when on $30 \%$ of spaced plants on plot at least single anthers were visible; 
- $\quad$ Estimated biomass yield (BY), in scale $1-9$, where 1 - the lowest yield, 9 - the highest yield;

- Length of longest stem incl. inflorescence / plant height (PH) [cm]

- $\quad$ Steam leaf length (LL) $[\mathrm{cm}]$,

- $\quad$ Steam leaf width (LW) [cm],

- $\quad$ Number of generative stems per plant (NGS);

- Inflorescence length (FL) [cm];

- $\quad$ Single inflorescence seed yield (SI) [g];

- $\quad$ Seed yield of single plant (SY) [g];

- $\quad$ Seed yield of plot (SP) $[\mathrm{kg}]$

- $\quad$ Thousand seed weight (TSW) [g]; determined according to ISTA rules (ISTA, 2015);

- $\quad$ Chlorophyll contents index (CCI) based on measurements made by CCM200 Plus on 3 steam leafs per plant, 5 measuring point per leaf from the base of leaf up to the top.

All calculations were made with STATISTICA ${ }^{\circledR}$ for Windows (Stat Soft, 2014) Significance of differences were accepted with $95 \%$ of probability. To identify the share of traits measured and observed in total variation of tested Festuca genotypes, Principal Component Analysis (PCA) was performed based on correlation matrix algorithm for all traits measured and averaged across years and locations. Multiple linear regression analysis with a forward stepwise variable selection procedure was used to develop a model for estimation of plant seed yield (SY) separately for each species. Data matrix from 2 years, 4 locations, 5 genotypes and 3 replications per genotype were used with all traits mentioned above, excluding SP.

\section{RESULTS AND DISCUSSION}

\section{Climatic conditions description}

Mean air temperatures during vegetation seasons were higher than normal values in all locations (Table 3 ). Total rainfall during vegetation season was lower than normal in both years in Radzików, as contrary to values measured in Leszno. Dry conditions in both years were noted in Nieznanice (from 128.1 to $218.5 \mathrm{~mm}$ less than normal for average rainfall during vegetation season) and Radzików (from 31.5 to $103.3 \mathrm{~mm}$ ). March and April were more wet than May $(20.1-22.5 \%$ more rain than normal), at June and July average rainfall was more or less similar to normal (107 $112 \%)$ and the end of growing season was usually dry (54-64\% of normal value). 
Climatic data (monthly means and normal values of air temperature and rainfall) for vegetation season at experiment locations.

\begin{tabular}{|c|c|c|c|c|c|c|c|c|c|}
\hline \multirow{2}{*}{ Month } & \multirow{2}{*}{$\begin{array}{c}\text { Values from } \\
\text { years: }\end{array}$} & \multicolumn{2}{|c|}{ Radzików } & \multicolumn{2}{|c|}{ Szelejewo } & \multicolumn{2}{|c|}{ Nieznanice } & \multicolumn{2}{|c|}{ Leszno } \\
\hline & & $\mathrm{T}$ & $\mathrm{R}]$ & $\mathrm{T}$ & $\mathrm{R}]$ & $\mathrm{T}$ & R] & $\mathrm{T}$ & R] \\
\hline \multirow{3}{*}{ March } & 2015 & 5.3 & 30.2 & 6.0 & 47.2 & 5.4 & 26.2 & 5.1 & 34.6 \\
\hline & 2016 & 4.2 & 38.4 & 4.4 & 64.2 & 4.5 & 37.9 & 4.5 & 55.3 \\
\hline & $1981-2000$ & 2.9 & 23.8 & 3.6 & 37.8 & 3.1 & 40.2 & 3.6 & 37.8 \\
\hline \multirow{3}{*}{ April } & 2015 & 8.4 & 38.0 & 9.1 & 44.0 & 9.0 & 31.4 & 8.7 & 45.3 \\
\hline & 2016 & 9.7 & 31.4 & 9.3 & 46.2 & 9.3 & 49.6 & 9.5 & 39.8 \\
\hline & $1981-2000$ & 8.9 & 30.0 & 8.8 & 31.3 & 8.7 & 46.4 & 8.8 & 31.3 \\
\hline \multirow{3}{*}{ May } & 2015 & 13.5 & 62.6 & 13.4 & 13.6 & 13.6 & 42.0 & 12.5 & 65.2 \\
\hline & 2016 & 16.0 & 31.6 & 15.5 & 30.4 & 14.9 & 39.2 & 15.4 & 35.4 \\
\hline & $1981-2000$ & 14.8 & 51.4 & 14.0 & 49.5 & 14.0 & 81.2 & 14.0 & 49.5 \\
\hline \multirow{3}{*}{ June } & 2015 & 17.5 & 25.2 & 16.6 & 50.2 & 17.2 & 51.2 & 15.0 & 80.0 \\
\hline & 2016 & 19.5 & 54.6 & 18.6 & 79.2 & 18.9 & 52.8 & 18.2 & 159.7 \\
\hline & $1981-2000$ & 17.4 & 60.0 & 16.6 & 56.8 & 16.8 & 86.4 & 16.6 & 56.8 \\
\hline \multirow{3}{*}{ July } & 2015 & 20.0 & 36.8 & 20.1 & 59.4 & 20.7 & 51.7 & 19.7 & 87.9 \\
\hline & 2016 & 19.8 & 89.8 & 19.6 & 116.8 & 19.5 & 104.1 & 18.8 & 120.9 \\
\hline & $1981-2000$ & 19.6 & 74.7 & 18.9 & 75.7 & 18.8 & 87.9 & 18.9 & 75.7 \\
\hline \multirow{3}{*}{ August } & 2015 & 22.6 & 4.8 & 22.8 & 68.8 & 22.7 & 19.8 & 22.5 & 58.1 \\
\hline & 2016 & 18.9 & 46.0 & 18.5 & 39.8 & 18.7 & 24.0 & 17.5 & 46.8 \\
\hline & $1981-2000$ & 18.9 & 50.8 & 18.3 & 60.8 & 18.1 & 75.7 & 18.3 & 60.8 \\
\hline \multirow{3}{*}{$\begin{array}{l}\text { Septem- } \\
\text { ber }\end{array}$} & 2015 & 15.3 & 31.4 & 15.2 & 29.8 & 15.4 & 39.4 & 14.5 & 31.4 \\
\hline & 2016 & 16.1 & 9.0 & 12.4 & 11.5 & 16.5 & 44.5 & 13.9 & 10.0 \\
\hline & $1981-2000$ & 13.7 & 41.5 & 13.7 & 41.7 & 13.5 & 62.4 & 13.7 & 41.7 \\
\hline \multirow{3}{*}{$\begin{array}{l}\text { Average/ } \\
\text { sum for: }\end{array}$} & III - IX 2015 & 14.7 & 229.0 & 14.7 & 313.0 & 14.9 & 261.7 & 14.0 & 402.5 \\
\hline & III - IX 2016 & 14.9 & 300.8 & 14.0 & 388.1 & 14.6 & 352.1 & 14.0 & 467.9 \\
\hline & $1981-2000$ & 13.7 & 332.3 & 13.4 & 353.6 & 13.3 & 480.2 & 13.4 & 353.6 \\
\hline
\end{tabular}

$\mathrm{T}$ - temperature $\left[{ }^{\circ} \mathrm{C}\right] ; \mathrm{R}$ - rainfall $[\mathrm{mm}]$

\section{Basic statistics, analysis of variance}

Majority of measured and observed phenotypic variables of three fescue species varied significantly among genotypes, study years and locations. Most of their interactions were also statistically significant (Table 4). Only for a few traits, no significant effect of different climatic conditions (years) was noticed: for tall fescue for BY and NGS, for meadow fescue - for OW and for red fescue - for FL. Significant effects of specific location conditions were calculated for all traits in all tested species. Moreover, significant effect of different genotypes was not calculated for: SI in meadow fescue and OW and HE for red fescue.

Significant effect of years for traits examined in perennial grasses is quite frequent and it results from the combination of different climatic conditions in succeeding years and age of plants (Elgersma 1990; Casler 1998; Fang et al. 2004; Fjellheim et al. 2007; Biligetu et al. 2012; Amini et al. 2013; Stukonis et al. 2015). It is also similar in case of locations, which were different in case of climatic conditions and soil characteristics. 


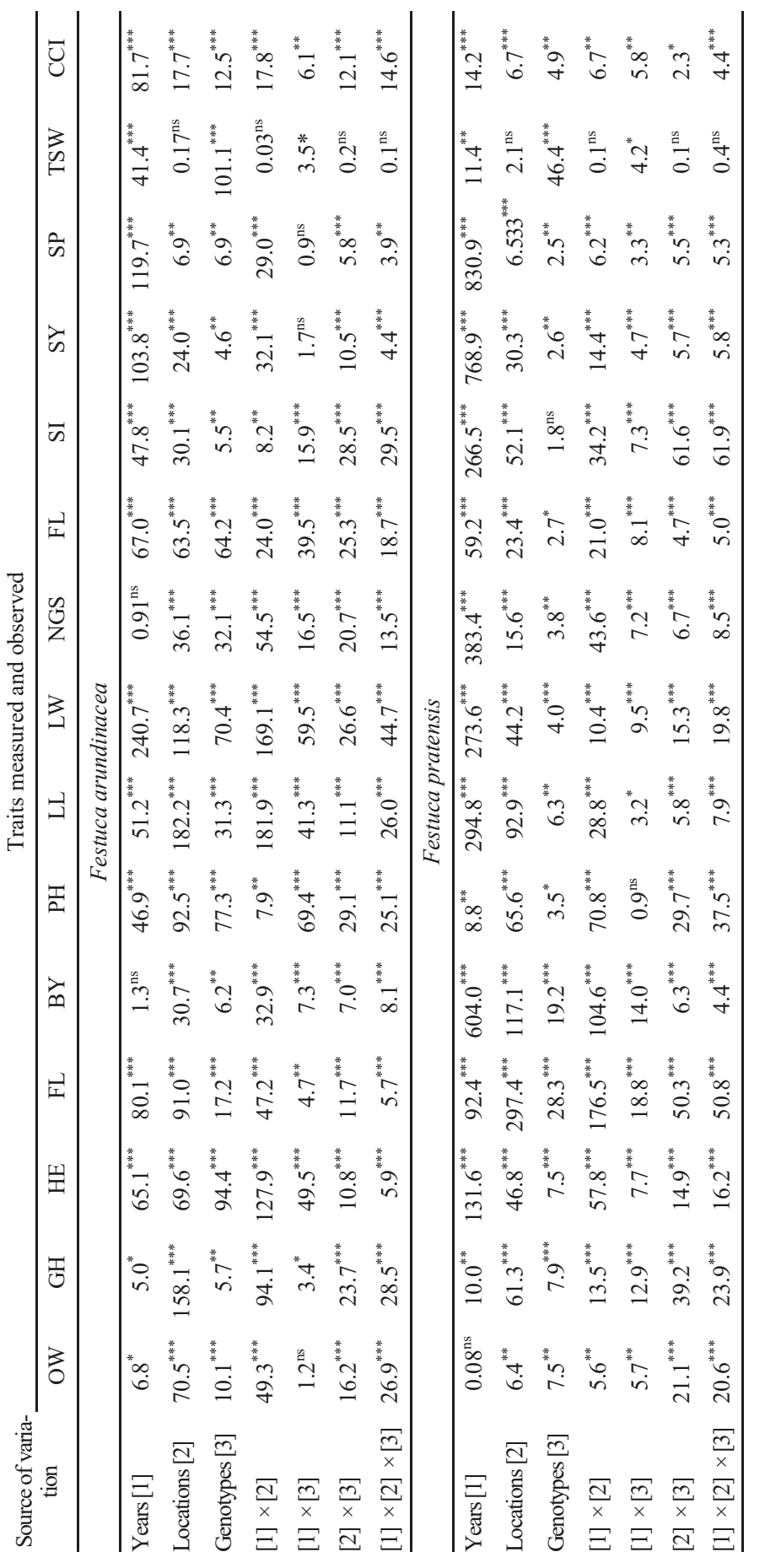


$\frac{\sqrt{\pi}}{\frac{\pi}{0}}$

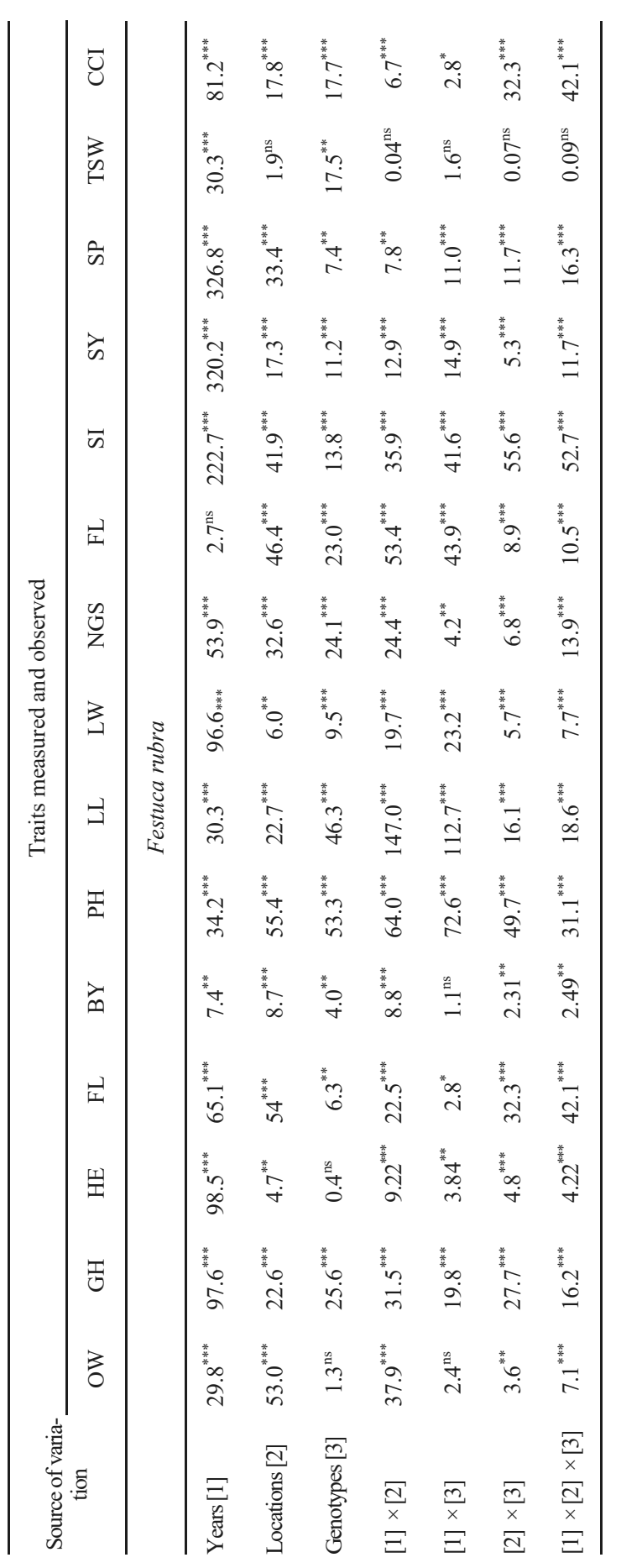


Variations of measured and observed traits were different in each species, but some similarities could be noticed (Table 5). The lowest values of coefficients on variation (V) were calculated for traits of known high heritability as HE, FE (Casler and Santen, 2010). High and very high values of $\mathrm{V}$ were calculated for seed yield traits (SI, SY, SP), NGS as well as for CCI.

Table 5

Mean values, standard errors (SE) and coefficients of variation (V\%) of seed production and other traits measured and observed on 15 genotypes from three Festuca species.

Values were averaged over 2 years, 4 locations and 3 replications

\begin{tabular}{|c|c|c|c|c|c|c|}
\hline \multirow{2}{*}{\multicolumn{2}{|c|}{ Trait/[unit] }} & \multicolumn{5}{|c|}{ Name or number of genotype: } \\
\hline & & Rahela & $121-2 / 8$ & $124-1 / 8$ & $127-1 / 1$ & $128-1 / 6$ \\
\hline \multicolumn{7}{|c|}{ Festuca arundinacea Schreb. } \\
\hline OW & mean & 8.2 & 7.8 & 7.7 & 7.8 & 7.5 \\
\hline [scale 1-9] & SE (V\%) & $\pm 0.31(10.7)$ & $\pm 0.41(15.0)$ & $\pm 0.42(15.4)$ & $\pm 0.47(16.9)$ & $\pm 0.41(15.6)$ \\
\hline $\mathrm{GH}$ & mean & 6.9 & 6.8 & 6.4 & 6.7 & 6.7 \\
\hline [scale 1-9] & SE (V\%) & $\pm 0.5(20.3)$ & $\pm 0.5(22.1)$ & $\pm 0.6(26.6)$ & $\pm 0.4(17.9)$ & $\pm 0.5(20.9)$ \\
\hline $\mathrm{HE}$ & mean & 41.1 & 45.6 & 46.4 & 47.0 & 49.3 \\
\hline [days] & $\mathrm{SE}(\mathrm{V} \%)$ & $\pm 1.5(10.2)$ & $\pm 2.1(12.7)$ & $\pm 1.0(6.3)$ & $\pm 1.9(11.5)$ & $\pm 1.8(10.3)$ \\
\hline $\mathrm{FE}$ & mean & 64.3 & 64.4 & 66.5 & 68.8 & 68.6 \\
\hline [days] & $\mathrm{SE}(\mathrm{V} \%)$ & $\pm 2.2(9.8)$ & $\pm 3.1(13.7)$ & $\pm 1.7(7.1)$ & $\pm 2.4(9.9)$ & $\pm 2.2(9.2)$ \\
\hline BY & mean & 8.1 & 8.1 & 7.8 & 7.9 & 7.8 \\
\hline [scale 1-9] & SE (V\%) & $\pm 0.21(7.4)$ & $\pm 0.20(6.2)$ & $\pm 0.19(7.7)$ & $\pm 0.23(7.6)$ & $\pm 0.31(9.0)$ \\
\hline PH & mean & 115.5 & 128.0 & 104.9 & 107.3 & 119.2 \\
\hline$[\mathrm{cm}]$ & $\mathrm{SE}(\mathrm{V} \%)$ & $\pm 4.73(11.6)$ & $\pm 6.64(14.7)$ & $\pm 7.28(19.6)$ & $\pm 5.90(15.6)$ & $\pm 7.18(17.0)$ \\
\hline LL & mean & 23.9 & 26.9 & 22.1 & 21.5 & 24.8 \\
\hline$[\mathrm{cm}]$ & $\mathrm{SE}(\mathrm{V} \%)$ & $\pm 2.21(25.9)$ & $\pm 2.58(27.1)$ & $\pm 2.83(36.2)$ & $\pm 2.07(27.0)$ & $\pm 3.6(41.1)$ \\
\hline LW & mean & 0.66 & 0.85 & 0.66 & 0.65 & 0.84 \\
\hline$[\mathrm{cm}]$ & $\mathrm{SE}(\mathrm{V} \%)$ & $\pm 0.09(42.9)$ & $\pm 0.07(22.2)$ & $\pm 0.13(57.1)$ & \pm 0.07 (28.6) & $\pm 0.12(37.5)$ \\
\hline NGS & mean & 133.5 & 85.5 & 140.7 & 154.3 & 100.5 \\
\hline [numbers] & $\mathrm{SE}(\mathrm{V} \%)$ & $\pm 26.9(57.0)$ & $\pm 13.46(44.6)$ & $\pm 25.81(51.9)$ & $\pm 28.12(51.5)$ & $\pm 20.01(56.3)$ \\
\hline FL & mean & 20.3 & 25.6 & 20.0 & 18.3 & 24.7 \\
\hline$[\mathrm{cm}]$ & $\mathrm{SE}(\mathrm{V} \%)$ & $\pm 2.35(32.5)$ & $\pm 1.89(20.7)$ & $\pm 2.78(39.5)$ & $\pm 0.79(12.0)$ & $\pm 2.26(25.9)$ \\
\hline SI & mean & 0.45 & 0.45 & 0.44 & 0.33 & 0.47 \\
\hline$[\mathrm{g}]$ & $\mathrm{SE}(\mathrm{V} \%)$ & $\pm 0.15(25.0)$ & $\pm 0.09(20.0)$ & $\pm 0.18(25.6)$ & $\pm 0.08(35.2)$ & $\pm 0.08(41.2)$ \\
\hline SY & mean & 29.0 & 26.5 & 29.9 & 33.1 & 32.5 \\
\hline$[\mathrm{g}]$ & $\mathrm{SE}(\mathrm{V} \%)$ & $\pm 5.41(52.8)$ & $\pm 4.51(48.3)$ & $\pm 4.09(38.8)$ & $\pm 3.91(33.5)$ & $\pm 4.62(40.3)$ \\
\hline SP & mean & 1.23 & 1.11 & 1.31 & 1.45 & 1.39 \\
\hline$[\mathrm{kg}]$ & SE (V\%) & $\pm 0.19(41.7)$ & \pm 0.14 & \pm 0.14 & $\pm 0.16(35.7)$ & $\pm 0.18(35.7)$ \\
\hline
\end{tabular}




\section{Continued}

Table 5

\begin{tabular}{|c|c|c|c|c|c|c|}
\hline \multirow{2}{*}{\multicolumn{2}{|c|}{ Trait/[unit] }} & \multicolumn{5}{|c|}{ Name or number of genotype: } \\
\hline & & \multirow[t]{2}{*}{ Rahela } & $121-2 / 8$ & \multirow{2}{*}{$\begin{array}{l}124-1 / 8 \\
\text { Schreb. }\end{array}$} & \multirow[t]{2}{*}{$127-1 / 1$} & \multirow[t]{2}{*}{$128-1 / 6$} \\
\hline \multicolumn{3}{|c|}{ Festuca arundinacea Schreb. } & & & & \\
\hline TSW & mean & 3.38 & 2.68 & 1.87 & 2.21 & 2.17 \\
\hline$[\mathrm{g}]$ & $\mathrm{SE}(\mathrm{V} \%)$ & $\pm 0.08(11.2)$ & $\pm 0.04(8.1)$ & $\pm 0.06(16.1)$ & $\pm 0.08(17.6)$ & $\pm 0.05(12.0)$ \\
\hline $\mathrm{CCI}$ & mean & 3.27 & 5.03 & 4.08 & 3.76 & 6.36 \\
\hline [unit] & $\mathrm{SE}(\mathrm{V} \%)$ & $\pm 0.77(66.7)$ & $\pm 1.17(66.0)$ & $\pm 1.42(97.6)$ & $\pm 0.87(65.8)$ & $\pm 2.09(92.2)$ \\
\hline \multicolumn{7}{|c|}{ Festuca pratensis Huds. } \\
\hline OW & mean & 6.8 & 6.9 & 6.6 & 6.7 & 7.3 \\
\hline [scale 1-9] & $\mathrm{SE}(\mathrm{V} \%)$ & $\pm 0.40(16.8)$ & $\pm 0.40(16.2)$ & $\pm 0.43(18.6)$ & $\pm 0.38(16.1)$ & $\pm 0.41(15.7)$ \\
\hline $\mathrm{GH}$ & mean & 5.9 & 5.2 & 5.5 & 5.8 & 5.2 \\
\hline [scale 1-9] & $\mathrm{SE}(\mathrm{V} \%)$ & $\pm 0.6(28.8)$ & $\pm 0.7(36.5)$ & $\pm 0.7(38.2)$ & $\pm 0.5(24.1)$ & $\pm 0.7(38.5)$ \\
\hline $\mathrm{HE}$ & mean & 51.6 & 51.9 & 51.8 & 50.7 & 51.8 \\
\hline [days] & SE (V\%) & $\pm 0.8(4.4)$ & $\pm 1.1(6.0)$ & $\pm 0.7(3.7)$ & $\pm 1.1(6.1)$ & $\pm 0.8(4.4)$ \\
\hline $\mathrm{FE}$ & mean & 63.5 & 65.5 & 65.8 & 64.4 & 66.1 \\
\hline [days] & $\mathrm{SE}(\mathrm{V} \%)$ & $\pm 2.4(10.9)$ & $\pm 1.6(6.9)$ & $\pm 1.5(6.5)$ & $\pm 2.0(8.5)$ & $\pm 1.5(6.7)$ \\
\hline BY & mean & 6.7 & 6.9 & 7.1 & 7.1 & 7.7 \\
\hline [scale 1-9] & SE (V\%) & $\pm 0.67(28.4)$ & $\pm 0.70(27.5)$ & $\pm 0.52(19.7)$ & $\pm 0.51(21.1)$ & $\pm 0.22(7.8)$ \\
\hline $\mathrm{PH}$ & mean & 96.3 & 100.4 & 97.5 & 98.7 & 99.4 \\
\hline$[\mathrm{cm}]$ & $\mathrm{SE}(\mathrm{V} \%)$ & $\pm 4.37(12.9)$ & $\pm 5.79(16.3)$ & $\pm 4.81(13.9)$ & $\pm 5.18(14.8)$ & $\pm 4.91(14.0)$ \\
\hline LL & mean & 19.3 & 20.0 & 19.4 & 18.5 & 18.7 \\
\hline$[\mathrm{cm}]$ & $\mathrm{SE}(\mathrm{V} \%)$ & $\pm 1.09(16.1)$ & $\pm 0.97(14.0)$ & $\pm 1.49(21.6)$ & $\pm 1.03(15.7)$ & $\pm 1.21(18.2)$ \\
\hline LW & mean & 0.67 & 0.68 & 0.65 & 0.66 & 0.62 \\
\hline$[\mathrm{cm}]$ & SE (V\%) & $\pm 0.06(28.6)$ & \pm 0.05 (14.3) & $\pm 0.04(14.3)$ & $\pm 0.06(28.6)$ & $\pm 0.06(33.3)$ \\
\hline NGS & mean & 97.5 & 104.7 & 105.1 & 114.1 & 123.5 \\
\hline [numbers] & SE (V\%) & \pm 21.34 (61.9) & $\pm 27.63(74.7)$ & $\pm 25.52(68.7)$ & $\pm 26.89(66.6)$ & $\pm 16.83(38.5)$ \\
\hline FL & mean & 19.4 & 19.8 & 19 & 18.9 & 18.5 \\
\hline$[\mathrm{cm}]$ & $\mathrm{SE}(\mathrm{V} \%)$ & $\pm 0.92(13.4)$ & $\pm 0.76(11.1)$ & $\pm 0.51(7.4)$ & \pm 0.89 (13.2) & $\pm 1.39(21.1)$ \\
\hline SI & mean & 0.20 & 0.21 & 0.22 & 0.20 & 0.20 \\
\hline$[\mathrm{g}]$ & SE (V\%) & $\pm 0.04(35.8)$ & $\pm 0.05(45.2)$ & $\pm 0.03(45.2)$ & $\pm 0.03(35.1)$ & $\pm 0.06(46.2)$ \\
\hline SY & mean & 15.7 & 18.8 & 17.3 & 16.1 & 17.4 \\
\hline$[\mathrm{g}]$ & $\mathrm{SE}(\mathrm{V} \%)$ & $\pm 4.02(72.6)$ & $\pm 5.26(79.3)$ & $\pm 4.33(71.1)$ & $\pm 3.67(64.6)$ & $\pm 3.55(57.5)$ \\
\hline SP & mean & 0.63 & 0.76 & 0.70 & 0.67 & 0.74 \\
\hline$[\mathrm{kg}]$ & $\mathrm{SE}(\mathrm{V} \%)$ & $\pm 0.17(83.3)$ & $\pm 0.22(75.0)$ & $\pm 0.17(71.4)$ & $\pm 0.14(57.1)$ & $\pm 0.16(71.4)$ \\
\hline TSW & mean & 2.24 & 2.37 & 2.29 & 2.24 & 2.33 \\
\hline$[\mathrm{g}]$ & $\mathrm{SE}(\mathrm{V} \%)$ & $\pm 0.03(7.1)$ & $\pm 0.03(6.1)$ & $\pm 0.08(18.0)$ & $\pm 0.05(11.5)$ & $\pm 0.04(8.5)$ \\
\hline $\mathrm{CCI}$ & mean & 4.12 & 4.84 & 4.01 & 4.3 & 3.84 \\
\hline [unit] & $\mathrm{SE}(\mathrm{V} \%)$ & $\pm 0.24(17.1)$ & $\pm 0.3(18.8)$ & $\pm 0.42(30.0)$ & $\pm 0.42(27.9)$ & $\pm 0.43(31.6)$ \\
\hline \multicolumn{7}{|c|}{ Festuca rubra L. } \\
\hline OW & mean & 7.8 & 7.7 & 7.9 & 7.6 & 8.0 \\
\hline [scale 1-9] & $\mathrm{SE}(\mathrm{V} \%)$ & $\pm 0.23(14.2)$ & $\pm 0.24(15.1)$ & $\pm 0.23(14.3)$ & $\pm 0.39(25.0)$ & $\pm 0.19(11.8)$ \\
\hline $\mathrm{GH}$ & mean & 5.4 & 5.5 & 6.4 & 5.8 & 6.9 \\
\hline [scale 1-9] & $\mathrm{SE}(\mathrm{V} \%)$ & $\pm 0.31(28.0)$ & $\pm 0.42(37.8)$ & $\pm 0.36(27.1)$ & $\pm 0.33(28.0)$ & $\pm 0.35(25.3)$ \\
\hline
\end{tabular}


Continued

Table 5

\begin{tabular}{|c|c|c|c|c|c|c|}
\hline \multirow{2}{*}{\multicolumn{2}{|c|}{ Trait/[unit] }} & \multicolumn{5}{|c|}{ Name or number of genotype: } \\
\hline & & \multirow[t]{2}{*}{ Rahela } & \multirow{2}{*}{\multicolumn{2}{|c|}{$\begin{array}{c}\text { 121-2/8 } \\
\text { Festuca rubra L. }\end{array}$}} & \multirow[t]{2}{*}{$127-1 / 1$} & \multirow[t]{2}{*}{$128-1 / 6$} \\
\hline & & & & & & \\
\hline $\mathrm{HE}$ & mean & 39.1 & 40.1 & 39.2 & 39.6 & 39.4 \\
\hline [days] & $\mathrm{SE}(\mathrm{V} \%)$ & \pm 0.85 (10.7) & $\pm 1.10(13.5)$ & $\pm 0.90(11.3)$ & \pm 0.84 & $\pm 1.39(17.3)$ \\
\hline $\mathrm{FE}$ & mean & 61.2 & 61.6 & 60.9 & 60.6 & 60.5 \\
\hline [days] & SE (V\%) & \pm 0.58 & $\pm 0.62(4.9)$ & \pm 0.59 (4.7) & \pm 0.64 & $\pm 0.61(5.0)$ \\
\hline BY & mean & 7.5 & 7.4 & 7.9 & 7.6 & 7.9 \\
\hline [scale 1- 9] & $\mathrm{SE}(\mathrm{V} \%)$ & $\pm 0.21(13.6)$ & $\pm 0.17(11.3)$ & $\pm 0.11(6.8)$ & $\pm 0.13(8.6)$ & $\pm 0.09(5.5)$ \\
\hline $\mathrm{PH}$ & mean & 83.8 & 70.7 & 81.1 & 80.7 & 78.0 \\
\hline$[\mathrm{cm}]$ & $\mathrm{SE}(\mathrm{V} \%)$ & $\pm 3.21(18.8)$ & $\pm 2.60(17.8)$ & $\pm 2.51(14.8)$ & $\pm 2.72(16.3)$ & $\pm 2.25(14.1)$ \\
\hline LL & mean & 14.0 & 10.9 & 12.9 & 11.6 & 12.3 \\
\hline$[\mathrm{cm}]$ & $\mathrm{SE}(\mathrm{V} \%)$ & $\pm 0.73(25.6)$ & $\pm 0.66(29.7)$ & $\pm 0.64(24.3)$ & $\pm 0.56(23.6)$ & \pm 0.45 (17.8) \\
\hline LW & mean & 0.28 & 0.24 & 0.26 & 0.23 & 0.27 \\
\hline$[\mathrm{cm}]$ & SE (V\%) & $\pm 0.01(15.3)$ & $\pm 0.02(32.0)$ & $\pm 0.01(19.5)$ & $\pm 0.02(36.5)$ & $\pm 0.01(27.0)$ \\
\hline NGS & mean & 253.5 & 283.0 & 332.2 & 343.8 & 421.4 \\
\hline [numbers] & SE (V\%) & $\pm 21.8(42.1)$ & $\pm 35.6(61.6)$ & $\pm 34.9(51.5)$ & $\pm 26.1(37.2)$ & $\pm 24.9(29.0)$ \\
\hline FL & mean & 14.8 & 12.5 & 14.1 & 12.7 & 13.3 \\
\hline$[\mathrm{cm}]$ & $\mathrm{SE}(\mathrm{V} \%)$ & $\pm 0.64(21.3)$ & $\pm 0.56(22.0)$ & $\pm 0.49(17.1)$ & $\pm 0.47(18.2)$ & \pm 0.45 (16.8) \\
\hline SI & mean & 0.15 & 0.15 & 0.13 & 0.10 & 0.13 \\
\hline$[\mathrm{g}]$ & $\mathrm{SE}(\mathrm{V} \%)$ & $\pm 0.016(52.9)$ & $\pm 0.032(49.2)$ & $\pm 0.014(55.4)$ & $\pm 0.010(49.9)$ & $\pm 0.022(58.1)$ \\
\hline SY & mean & 23.4 & 22.7 & 27.0 & 23.0 & 29.1 \\
\hline$[\mathrm{g}]$ & $\mathrm{SE}(\mathrm{V} \%)$ & \pm 2.14 (44.7) & $\pm 2.78(60.0)$ & $\pm 1.98(35.9)$ & $\pm 2.00(42.6)$ & $\pm 1.88(31.7)$ \\
\hline SP & mean & 1.11 & 0.98 & 1.16 & 1.03 & 1.26 \\
\hline$[\mathrm{kg}]$ & SE (V\%) & $\pm 0.11(50.8)$ & $\pm 0.13(66.5)$ & $\pm 0.11(46.2)$ & $\pm 0.09(45.0)$ & $\pm 0.12(46.1)$ \\
\hline TSW & mean & 1.37 & 1.10 & 1.23 & 0.82 & 1.14 \\
\hline$[\mathrm{g}]$ & $\mathrm{SE}(\mathrm{V} \%)$ & $\pm 0.06(22.9)$ & $\pm 0.04(16.0)$ & $\pm 0.05(19.2)$ & $\pm 0.02(13.0)$ & $\pm 0.03(14.4)$ \\
\hline $\mathrm{CCI}$ & mean & 2.2 & 1.7 & 1.8 & 2.3 & 2.0 \\
\hline [unit] & $\mathrm{SE}(\mathrm{V} \%)$ & $\pm 0.49(87.7)$ & $\pm 0.19(45.6)$ & $\pm 0.29(60.4)$ & $\pm 0.58(98.8)$ & $\pm 0.41(79.8)$ \\
\hline
\end{tabular}

\section{Principal Component Analysis}

The first two factors of the PCA based on phenotypic traits explained from $86.4 \%$ (meadow fescue) to $90.7 \%$ (tall fescue) of variation present in tested material, respectively (Table 6). First factors calculated for all species were significantly correlated with development of generative-related traits as NGS, FL and single panicle seed yield (SI). However, only for meadow and tall fescue first factors were also significantly correlated with some vegetative-related traits as PH, LL, LW and CCI. 
Results of Principal Component Analysis. Values of correlation coefficients between the Principal Components and the traits examined were shown in table. Values above 0.7 were statistically significant

\begin{tabular}{|c|c|c|c|c|c|c|c|c|c|}
\hline \multirow{3}{*}{ Trait: } & \multicolumn{3}{|c|}{ Festuca arundinacea } & \multicolumn{3}{|c|}{ Festuca pratensis } & \multicolumn{3}{|c|}{ Festuca rubra } \\
\hline & \multicolumn{3}{|c|}{ Factor no. } & \multicolumn{3}{|c|}{ Factor no. } & \multicolumn{3}{|c|}{ Factor no. } \\
\hline & 1 & 2 & 3 & 1 & 2 & 3 & 1 & 2 & 3 \\
\hline OW & -0.05 & -0.32 & -0.95 & -0.73 & 0.39 & 0.56 & 0.34 & 0.93 & -0.03 \\
\hline $\mathrm{GH}$ & 0.36 & -0.81 & -0.42 & 0.53 & -0.74 & -0.38 & -0.08 & 0.94 & 0.13 \\
\hline $\mathrm{HE}$ & 0.50 & 0.74 & 0.44 & -0.04 & 0.91 & 0.21 & -0.37 & -0.76 & -0.43 \\
\hline $\mathrm{FE}$ & 0.10 & 0.95 & 0.31 & -0.10 & 0.97 & 0.11 & 0.92 & -0.02 & 0.26 \\
\hline BY & 0.77 & -0.21 & -0.53 & -0.58 & 0.40 & 0.63 & -0.41 & 0.84 & -0.35 \\
\hline $\mathrm{PH}$ & 0.90 & -0.37 & -0.21 & 0.74 & 0.16 & -0.58 & 0.50 & 0.65 & 0.54 \\
\hline LL & 0.95 & -0.24 & -0.17 & 0.75 & -0.18 & -0.60 & 0.68 & 0.62 & 0.39 \\
\hline LW & 0.99 & 0.03 & 0.09 & 0.78 & -0.20 & -0.59 & 0.69 & 0.66 & 0.14 \\
\hline NGS & -0.93 & 0.27 & 0.14 & -0.84 & 0.26 & 0.45 & -0.94 & 0.32 & 0.10 \\
\hline SI & 0.97 & 0.07 & 0.07 & 0.96 & -0.21 & 0.07 & 0.93 & 0.26 & 0.22 \\
\hline SY & -0.49 & 0.86 & -0.12 & -0.38 & 0.15 & 0.91 & 0.04 & 0.99 & -0.10 \\
\hline SP & -0.66 & 0.75 & 0.03 & -0.08 & 0.31 & 0.92 & 0.22 & 0.96 & -0.04 \\
\hline FL & 0.99 & -0.04 & 0.14 & 0.95 & 0.00 & -0.30 & 0.82 & 0.51 & 0.27 \\
\hline TSW & -0.03 & 0.80 & 0.35 & -0.56 & 0.17 & 0.45 & 0.74 & 0.05 & 0.02 \\
\hline CCI & 0.96 & -0.14 & 0.23 & 0.55 & -0.36 & -0.69 & 0.21 & -0.17 & 0.96 \\
\hline$\% \mathrm{VE}$ & 62.7 & 28.0 & 7.4 & 71.4 & 15.0 & 8.8 & 59.4 & 29.0 & 8.4 \\
\hline
\end{tabular}

$\mathrm{VE}-\%$ of variation explained

Second factors, accounting from $15 \%$ (meadowl fescue) to $29 \%$ (red fescue) of total variation, were significantly related to seed yield traits (SY, SP) for tall and red fescue and also with GH and HE for all three species. Only for meadow fescue, seed yield traits (SY and SP) were significantly correlated with third factor, that accounts only for $8.8 \%$ of total variation. Distribution of loadings significantly related to factors 1 and 2 were similar for all three tested species in case of generative-related traits. But in case of vegetative-related traits it was similar only for tall and meadow fescues.

\section{Pearson's correlation coefficients}

Simple correlations were calculated separately for each species. However, in case of some traits it was similar for all Festuca species tested (Table 7).

For mean flowering start date (FE) significant and positive correlation coefficients were calculated with OW for all three species. It means that OW highranked genotypes produced flowers later. Not such relation was calculated for $\mathrm{OW}$ and HE, immediately preceding flowering, but for the later there was no significant correlation with $\mathrm{FE}$, excl. red fescue. 


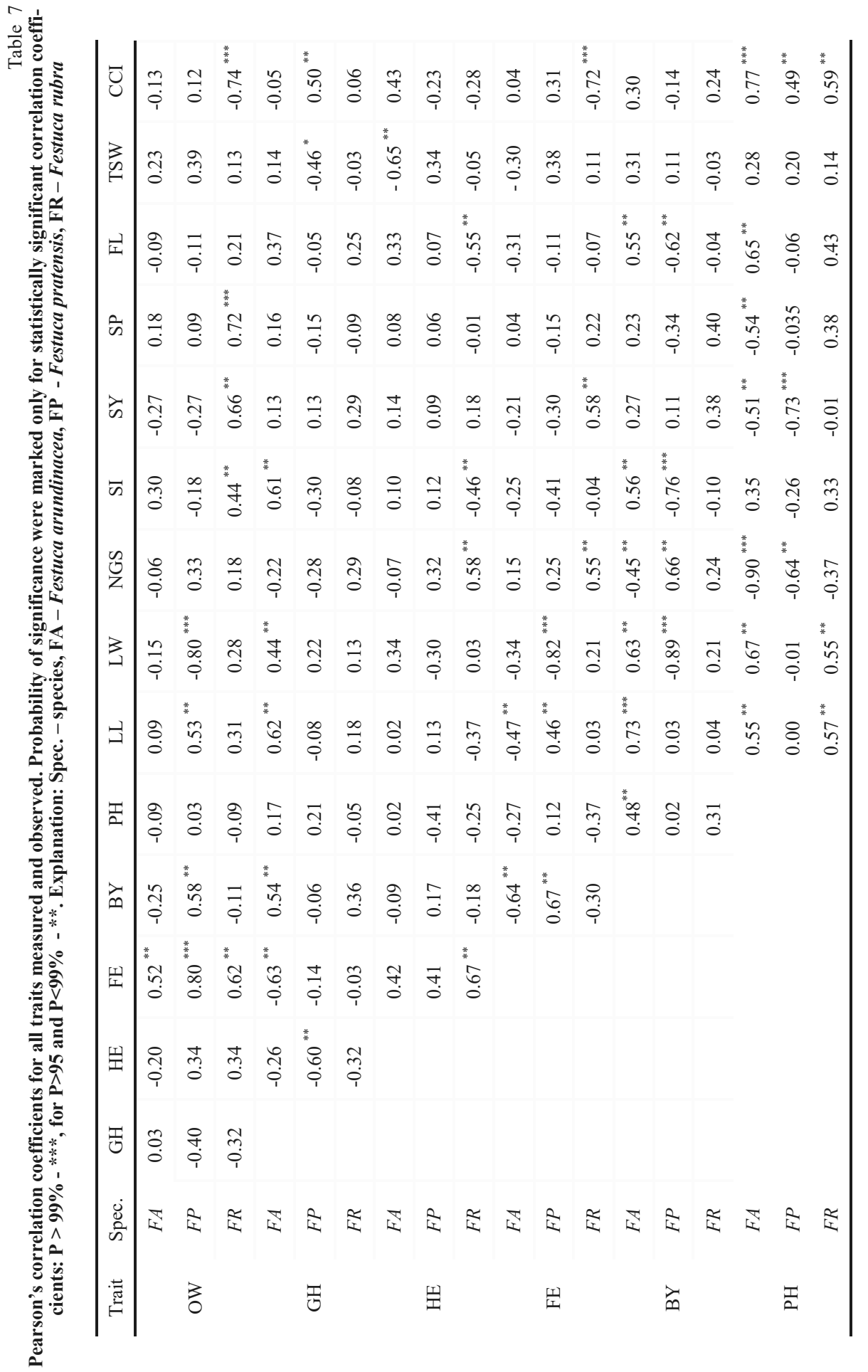




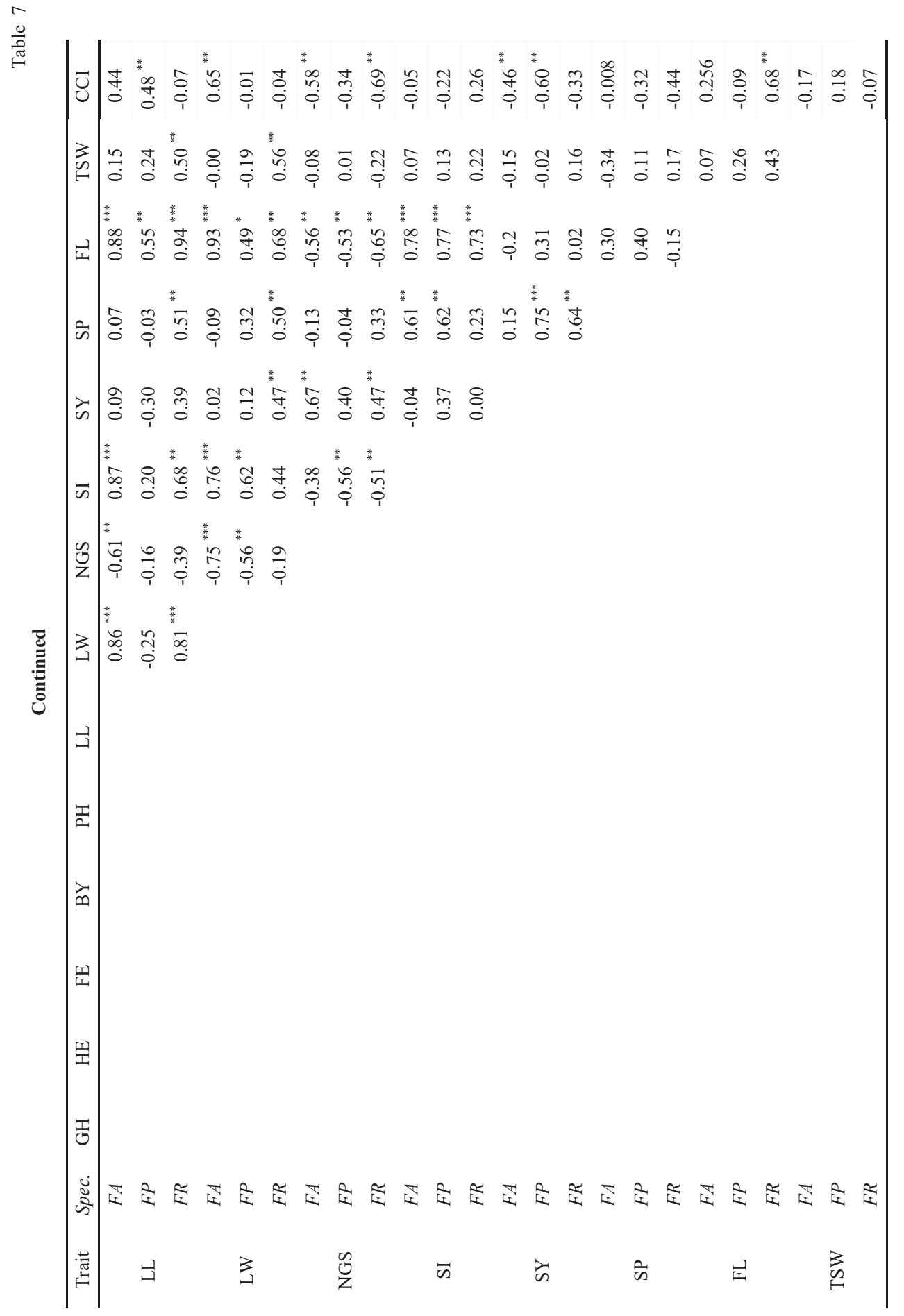


For PH and CCI significant and positive correlation coefficients were also calculated for all species. For important component of seed yield production, i.e. length of the seed head - FL, significant and positive correlation coefficients were calculated for LL, LW, and SI. For NGS correlation was significant but negative. If steam density increases, seed heads become smaller and shorter, as a consequence of decreasing space (Fairey and Lefkovitch 1999). It has been described by Mäkelä and Kousa (2009) that meadow fescue have good ability to compensate among the components of seed yield. Lower number of panicles was compensated with increased panicle size. The number of reproductive tillers per unit area is an important component in establishing the seed yield potential; however, its importance varies among grass species and it is of low heritability (Boelt and Studer, 2010).

\section{Seed yield prediction}

For regression analysis we used both vegetative-related traits (as $\mathrm{OW}, \mathrm{GH}$, BY, CCI, LL, LW) and traits close related to generative phase of plant development (PH, HE, FE, FL, NGS, TSW).

Multiple regression analysis with backward stepwise selection resulted in different numbers of SY predictors for different species (Table 8). For meadow fescue five, for red fescue three and for tall fescue - only two statistically significant predictors were selected. For all species LW and NGS were statistically significant and had positive weights. For meadow and red fescue - also SI was significant with positive weight. Only for meadow fescue OW with negative weight and BY (with positive) were accounted also as traits explaining SY variation.

Table 8

Results of multiple regression analysis with SY (seed yield per plant) as dependent variable. Standardized $\left(b^{*}\right)$, unstandardized (b) coefficients and probability of significance $(P)$ of predictors were shown

\begin{tabular}{|c|c|c|c|c|c|c|c|c|c|}
\hline \multirow{2}{*}{ Model/ Trait } & \multicolumn{3}{|c|}{ Festuca arundinacea } & \multicolumn{3}{|c|}{ Festuca pratensis } & \multicolumn{3}{|c|}{ Festuca rubra } \\
\hline & $\mathbf{b} *$ & b & $\mathbf{P}$ & b * & b & $\mathbf{P}$ & b * & b & $\mathbf{P}$ \\
\hline Constant & - & -3.72 & n.s. & - & -15.6 & $* * *$ & - & 12.7 & $* * *$ \\
\hline LW & 0.50 & 23.60 & $* * *$ & 0.27 & 20.14 & $* * *$ & 0.32 & 37.24 & $* * *$ \\
\hline NGS & 0.72 & 0.13 & $* * *$ & 0.62 & 0.11 & $* * *$ & 0.79 & 0.05 & $* * *$ \\
\hline SI & - & - & - & 0.19 & 19.34 & $* * *$ & 0.50 & 85.75 & $* * *$ \\
\hline OW & - & - & - & -0.18 & -1.16 & $* *$ & - & - & - \\
\hline BY & - & - & - & 0.29 & 2.25 & $* * *$ & - & - & - \\
\hline $\mathrm{R}$ & 0.63 & & & 0.92 & & & 0.83 & & \\
\hline $\mathrm{R}^{2}$ adjusted & 0.39 & & & 0.83 & & & 0.68 & & \\
\hline SE & 10.4 & & & 4.77 & & & 4.57 & & \\
\hline
\end{tabular}

Explanations: $* * *$ - for $\mathrm{P}>99,9 \%, * *$ - for $99,9 \%>\mathrm{P}>95 \%(* *)$, n.s. - not significant; $\mathrm{SE}-\mathrm{SE}$ of estimation

Results of the multiple regression analysis indicate that in all species NGS had the relatively greatest contribution in the prediction of variation in SY. It has been also confirmed by Nguyen and Sleper (1983) that number of fertile tillers had the largest effect on seed yield of tall fescue. Next traits, by means of relative contribution in prediction of SY variation were LW (for tall fescue) and BY and LW (for meadow fescue) and SI and LW (for red fescue). Considering 
multiple regression analysis, the size of the beta factors $\left(b^{*}, b\right)$, allows to compare the relative contributions that each independent variable makes in the prediction of dependent variable (Rawlings et al. 1998). However, care must be used on the interpretation of results, that multiple regression analysis has established only that variation in seed yield per plant (SY) was associated with variation in LW, NGS and other traits as listed in tab. 8. (Rawlings at al. 1998). Thus, it would be incorrect to conclude that LW, NGS and other traits are important casual variables of SY.

Positive and relatively high contribution of LW has been also described by Fang et al. (2004) for meadow fescue. Steam and flag leaves produce substantial amount of assimilates transported to the ear after anthesis. These authors demonstrated that leaf width has an important direct effect on seed yield and an indirect one through panicle fertility, indicates that large leaves contribute to a good seed-set (panicle fertility) through assimilate reallocation via the stems to the inflorescence in the period of anthesis, and that this contribute to higher seed yields. It is well known phenomenon for cereals, and it is not surprising that this is the case also in grasses.

Conclusion from our experiments is that despite observed differences between tested species, similar measures could be applied to describe seed yield variation. Breeders should not only select for increased size of reproductive system but also for some vegetative traits, with possible enhancement of forage production.

\section{ACKNOWLEDGEMENTS}

Above work has been financially supported by The Ministry of Agriculture and Rural Development (Basic Research for Biological Progress in Plant Production, 2014 - 2016, task no. 38).

\section{REFERENCES}

Amini F., Majid M.M., Mirlohi A. 2013. Genetic and genotype x environment interaction analysis for agronomical and some morphological traits in half-sib families of tall fescue. Crop Sci. 53, $411-421$.

Biligetu B., Schellenberg M.P., McLeod J.G., Wang Z. 2012. Seed yield variation in plains rough fescue (Festuca halli (Vasey) Piper) populations and its relation with phenotypic characteristics and environmental factors. Grass and Forage Science. 68: 589 - 595.

Boelt B., Studer B. 2010. Breeding for grass seed yield. In: Boller B., Posselt U. K., Veronesi F. (eds.) Fodder crops and amenity grasses. Handbook of Plant Breeding, vl. 5., Springer Science+Business Media, LLC, New York, Dordrecht, Heidelberg, London, $161-174$.

Casler M.D. 1998. Genetic variation within eight populations of perennial forage grasses. Plant Breeding 117: $243-249$.

Casler M.D., Santen E. 2010. Breeding objectives in forages. In: Boller B., Posselt U. K., Veronesi F. (eds.) Fodder crops and amenity grasses. Handbook of Plant Breeding, vl. 5., Springer Science+Business Media, LLC, New York, Dordrecht, Heidelberg, London, 115 - 136.

Danielewicz D., Surma- Ślusarska B., Żurek G., Martyniak D., Kmiotek M., Dybka K. 2015. Selected grass plants as biomass fuels and raw materials for papermaking, part II. Pulp and paper properties. BioResources 10(4): $8552-8564$

El Bassam N. 2010, Handbook of Bioenergy Crops, Earthscan, 1 - 516.

Elgersma A. 1990. Seed yield related to crop development and to yield components in nine cultivars of perennial ryegrass (Lolium perenne L.). Euphytica 49: 141 - 154.

Fang C., Aamlid T.S., Jørgensen Ø., Rognli O.A. 2004. Phenotypic and genotypic variation in seed production traits within a full-sib family of meadow fescue. Plant Breeding 123, $241-246$. 
Fairey, N. A., Lefkovitch, L. P. 1999. Crop density and seed production of tall fescue (Festuca arundinacea Schreber). 2. Reproductive components and seed characteristics. Can. J. Plant Sci. 79: 543-549.

Fjellheim S., Rognli O.A., Fosnes K., Brochman C. 2006. Phylogeographical history of the widespread meadow fescue (Festuca pratensis Huds.) inferred from chloroplast DNA sequences. J. Biogeography, 33: $1470-1478$.

Fjellheim S., Blomlie A.B., Marum P., Rogli O.A. 2007. Phenotypic variation in local populations and cultivars of meadow fescue - potential for improving cultivars by utilizing wild germplasm. Palnt Breeding $126 ; 279-286$.

Griffiths D. J., Lewis J., Bean E.W. 1980. Problems of breeding for seed production in grasses. In: P.D. Hebblethwaite (ed.) Seed Production. Butterworths, London - Boston. 37 - 49.

Hill, M. J., and B. R. Watkin, 1975: Seed production studies on perennial ryegrass, timothy and prairie grass. 1: Effect of tiller age on tiller survival, ear emergence and seedhead components. J. Br. Grassld Soc. 30, $63-71$.

ISTA, International Seed Testing Association, 2015. International Rules for Seed Testing, vol. 2015,10-1,

Lalak J., Martyniak D., Kasprzycka A., Żurek G., Moroń W., Chmielewska M., Wiącek D., Tys J. 2016. Comparison of selected parameters of biomass and coal. International Agrophysics, 30: 475 - 482.

Markgraf-Dannanberg I. 1980. Festuca. In: Tutin T.G., Heywood V.H., Burges N.A., Moore D.M., Valentine D.H., Walters S.M., Webb D.A. (eds.) Flora Europea, vol. 5. Alismataceae to Orchidaceae (Monocotyledones), Cambridge University Press, $125-153$.

Mäkelä P., Kousa M. 2009. Seed production of two meadow fescue cultivars differing in growth habit. Agricultural and Food Science, 18: 91 - 99.

Martyniak D., Żurek G., Prokopiuk K. 2017. Biomass yield and quality of wild populations of tall wheatgrass [Elymus elongatus (Host.) Runemark], Biomass \& Bioenergy, 101: $21-29$.

Meyer W.A., Watkins E. 2003. Tall Fescue (Festuca arundinacea). In: Casler M.D., Duncan R.R. (eds.) Turfgrass biology, genetics and breeding. John Wiley \& Sons, Inc. Hoboken, New Jersey, USA, 107 127.

Nguyen H.T., Sleper D.A. 1983. Genetic variability of seed yield and reproductive characters in tall fescue. Crop Science, 23 (4): $621-626$.

Rawlings J.O., Pantula S. G., Dickey D.A., 1998. Applied Regression Analysis. A research tool. Second Edition. Springer Verlag New York, Inc. pp. 671.

Rognli, O.A. 2007. Genetic analysis of seed yield components. In: Procc.of the XXVII'th EUCARPIA Symposium on Improvement of Fodder Crops and Amenity Grasses. Denmark, 19 - 23.08.2007, Copenhagen, pp. $83-87$.

Rognli O.A., Saha C.M., Bhamidimarri S., Heijden , S. van der, 2010. Fescues. In: Boller B., Posselt U.K., Veronesi F. (eds.) Fodder Crops and Amenity Grasses. Handbook of Plant Breeding, vol. 5. Springer Science+Business Media, LLC 2010, 261 - 292.

Ruemmele B.A., Wipff J. K., Brilman L., Hignight K.W. 2003. Fine - leaved Festuca species. In: Casler M.D., Duncan R.R. (eds.) Turfgrass biology, genetics and breeding. John Wiley \& Sons, Inc. Hoboken, New Jersey, USA, $129-174$.

Sleper D.A., West C.P. 1996. Tall Fescue. In: Moser L.E., Buxton D.R., Casler M.D. (eds.) Cool-Season Forage Grasses, Agronomy Monograph no. 34, Am. Soc. of Agronomy, Inc. Madison, Wisconsin, USA, $471-502$.

Stat Soft, Inc. 2014. STATISTICA (data analysis software system), ver. 12, www.statsoft.com

Stukonis V., Juzenas S., Ceseviciene J., Norkeviciene E. 2015. Assesment of morpho-anatomical traits of red fescue (Festuca rubra L.) germplasm differing in origin. Zemdirbyste-Agriculture, 102(4); 437 - 442.

Wang Q., Hu T., Cui J., Wang X., Zhou H., Han J., Zhang T. 2011. Modelling of seed yield and its components in tall fescue (Festuca arundiancea) based on a large sample. African Journ. of Biotechnology, 10 (59); $12584-12594$. 